\title{
Esplenectomía en hematología: ¿sigue teniendo lugar en el manejo de la trombocitopenia inmune primaria?
}

Splenectomy in hematology: Does it still have a place in the management of primary immune thrombocytopenia?

\footnotetext{
- Carmen Rosales, ${ }^{1,2}$, Virginia Abello,

'Departamento de Hematología y Unidad de Trasplante de Médula Ósea, Clínica de Marly (Bogotá, Colombia). ${ }^{2}$ Hospital de San José, Fundación Universitaria de Ciencias de la Salud (FUCS) (Bogotá, Colombia).
}

La esplenectomía hace parte del armamentario diagnóstico y terapéutico de un sinnúmero de patologías hematológicas. A través del tiempo, sus indicaciones han variado, más con base en el sentido común y en la práctica clínica rutinaria que sobre la mejor evidencia. Estos cambios también han sido motivados por el progreso en el manejo de las enfermedades malignas y no malignas, por la introducción de nuevas estrategias diagnósticas y por la modificación de la morbimortalidad relacionada con el procedimiento quirúrgico. Desde el punto de vista diagnóstico, la esplenectomía es de especial importancia en los síndromes linfoproliferativos, donde en algunas ocasiones la esplenomegalia es la principal manifestación de la enfermedad' ${ }^{1}$.

Tabla 1. Indicaciones de esplenectomía en enfermedades hematológicas ${ }^{1}$

Trombocitopenia inmune primaria crónica Trombocitopenia relacionada a VIH

Esferocitosis hereditaria

Enfermedad de Hodgkin

Anemia hemolítica autoinmune

Linfoma no Hodgkin

Leucemia linfoide crónica

Desórdenes mieloproliferativos crónicos

Leucemia de células peludas

Anemia de células falciformes

Beta-talasemia
En este número, Acevedo y colaboradores revisan una serie de 30 pacientes esplenectomizados por patologías hematológicas neoplásicas o no (en un $60 \%$ de ellos la indicación fue precisamente esa). La trombocitopenia inmune primaria (TIP) es la indicación más frecuente de esplenectomía terapéutica en adultos, intervención que ha sido el pilar de manejo de esta entidad desde hace más de 50 años, incluso antes de la introducción de los esteroides como terapia de primera línea. Después del advenimiento de los corticoides, la cirugía se reserva para pacientes refractarios o en quienes dependen de ellos por más de seis meses para mantener un recuento plaquetario seguro. En una revisión sistemática publicada en el año 2004, se estableció que la tasa de remisión completa (> 150.000 plaquetas mantenidas sin tratamiento adicional) en 2.633 pacientes adultos fue del $66 \%$ y la tasa de remisión completa o parcial (> 30 o 50.000 plaquetas, por lo menos por 30 días, con o sin otro tratamiento) fue del $88 \%$. La mediana de recaída en 48 estudios que evaluaron este desenlace fue del 15\% (rango 0-51\%) con una mediana de seguimiento de 33 meses $^{2}$. En línea con estos resultados en la serie que nos ocupa, la respuesta de los pacientes con TIP a la esplenectomía fue del $80 \%$, sin ninguna recaída.

A pesar de los resultados relativamente buenos de la esplenectomía, han aparecido otros tratamientos que se pueden considerar hoy en día en una segunda línea, y tanto los médicos como algunos pacientes pueden sentirse incómodos ante la opción de un tratamiento quirúrgico, así la tasa de complicaciones sea relativamente baja (12.9\% con laparotomía y $9.6 \%$ con laparoscopia) y la mortalidad menor al $1 \%^{2}$; en esta serie, no hubo ninguna muerte temprana relacionada con el procedimiento.

Además de las tasas de respuesta, al decidir sobre la segunda línea, se deben tener en cuenta otras variables, como los eventos adversos, el costo, el impacto en la calidad de vida y las preferencias del paciente. En segunda línea, se consideraban hasta el año 2010 la azatioprina, vincristina, dapsona, danazol, micofenolato mofetil y ciclofosfamida; cada uno de ellos con un perfil de seguridad y eficacia variable. La investigación con estos agentes en esta indicación es escasa, por lo cual es difícil hacer recomendaciones de uno sobre el otro o acerca del orden en que se deben utilizar. En la mayoría de los casos, la elección se define por la experiencia 
personal del hematólogo. En los últimos años, dos nuevos tipos de agentes han despertado gran interés en la comunidad científica: los agonistas del receptor de la trombopoyetina (romiplostin y eltrombopag) que han demostrado ser efectivos en pacientes con trombocitopenia persistente esplenectomizados o no. Las tasas de respuesta plaquetaria con estos agentes oscilan entre el $70 \%$ y $80 \%$, requiriendo su uso crónico, y, en algunos casos, al suspenderlos, la trombocitopenia puede no solo reaparecer, sino empeorar. En general, son bien tolerados, siendo el aumento de las transaminasas y de la trama reticulínica en la médula ósea los eventos adversos más significativos, pero con una relevancia clínica que está por establecerse ${ }^{3-5}$.

El otro agente que ha despertado interés es el rituximab; una revisión sistemática de la literatura dirigida hacia este tema mostró una respuesta global del $62.5 \%$, resultado que puede ser duradero. Sin embargo, después de un año, solo el $18 \%$ al $35 \%$ de los pacientes mantiene el beneficio, aunque no todos requieren una nueva intervención ${ }^{6}$. Se han reportado complicaciones severas que amenazan la vida en el
3.3\% de los pacientes, con una mortalidad estimada cercana al $3 \%$. Recientemente, se han reportado casos de leucoencefalopatía multifocal como complicación del uso de rituximab.

En conclusión, hay una gran cantidad de agentes que pueden ser utilizados en segunda línea y no hay ninguna evidencia que permita guiar la secuencia de tratamiento. La esplenectomía continúa siendo la única alternativa que permite ofrecer respuestas completas duraderas sin tratamiento en una proporción significativa de pacientes. Ante lo escaso de la información disponible, esta serie de 30 pacientes, en un solo centro, representa una descripción valiosa de la esplenectomía en indicaciones hematológicas en nuestro medio. Aunque se trata de un grupo heterogéneo de personas, con indicaciones y patologías diferentes, incluye un número significativo de pacientes con TIP, en los cuales se confirma una efectividad y seguridad similar a lo reportado en la literatura mundial, que puede ser útil en el momento de decidir la segunda línea de tratamiento en un paciente con una respuesta inadecuada a corticoides.

\section{Referencias}

1. Baccarani U, Terrosu G, Donini A, Zaja F, Bresadola F, Baccarani M. Splenectomy in hematology. Current practice and new perspectives. Haematologica. 1999;84(5):431-6.

2. Kojouri K, Vesely SK, Terrell DR, George JN. Splenectomy for adult patients with idiopathic thrombocytopenic purpura: a systematic review to assess long-term platelet count responses, prediction of response, and surgical complications. Blood. 2004;104(9):2623-34.

3. Kuter DJ, Bussel JB, Lyons RM, Pullarkat V, Gernsheimer TB, Senecal FM, et al. Eficacy of romiplostim in patients with chronic immune thrombocytopenic purpura: a double-blind randomised controlled trial. Lancet. 2008;371(9610):395-403.
4. Bussel JB, Kuter DJ, Pullarkat V, Lyons RM, Guo M, Nichol JL. Safety and efficacy of long-term treatment with romiplostim in thrombocytopenic patients with chronic ITP. Blood. 2009;113(10):2161-71.

5. Bussel JB, Provan D, Shamsi T, Cheng G, Psaila B, Kovaleva L, et al. Effect of eltrombopag on platelet counts and bleeding during treatment of chronic idiopathic thrombocytopenic purpura: a randomised, double-blind, placebo-controlled trial. Lancet. 2009;373(9664):641-8.

6. Arnold DM, Dentali F, Crowther MA, Meyer RM, Cook RJ, Sigouin $C$, et al. Systematic review: efficacy and safety of rituximab for adults with idiopathic thrombocytopenic purpura. Ann Intern Med. 2007;146(1):25-33. 